\title{
Un nuevo enfoque predictivo de la fragmentación en la Voladura de Rocas
}

\begin{abstract}
RESUMEN
La Importancia del presente trabajo de investigación es proporcionar un nuevo enfoque predictivo de la fragmentación en los Procesos de Voladura de Rocas, desarrollado en base al modelo KUZ-RAM, técnicas de Análisis Multivariante (MVA) y técnicas de Redes Neuronales Artificiales (ANN).

El objetivo del nuevo enfoque predictivo de la fragmentación se sustenta en que dicha investigación nos proporcionaría una optimización en las operaciones minero-metalúrgicas, porque entregando una óptima fragmentación requerida para los procesos de Molienda, se podría minimizar sus tiempos y maximizar su productividad.
\end{abstract}

Palabras-claves: Enfoque; predictivo; ingenieria; fragmentación; voladura de rocas; optimización

A NEW APPROACH TO PREDICTIVE OF FRAGMENTATION IN THE BLAST OF ROCKS

\section{ABSTRACT}

The importance of this research work is to provide a new predictive approach of the fragmentation in the blasting of rocks processes, developed based on the KUZ-RAM model, Multivariate Analysis techniques (MVA) and Artificial Neural Network techniques (ANN)

The objective of the new predictive approach to fragmentation is based on the fact that this research would provide us an optimization in the metallurgical mining operations, because delivering an optimal fragmentation requerid for the grinding processes it could minimize their times and maximize their productivity.

Keywords: Approach; predictive; engineering; fragmentation; rock blasting; optimization.

\section{INTRODUCCIÓN}

El Anuario Minero del Ministerio de Energía y Minas del Perú (2016) sostiene que el elemento dinámico en la economía peruana, es la inversión privada, que represento al cierre del año 2016 un PBI de $17.7 \%$ siendo este un PBI real equivalente a 3.9\%. La inversión empresarial se focaliza en actividades económicas en las cuales el Perú destaca a nivel mundial como minería, pesca, agro exportación y turismo. Según información de Pro inversión, la proporción mayor del saldo de Inversión Extranjera Directa (IED) al cierre del año 2016, correspondió al sector minero (22.88\%), seguido de comunicaciones (19.75\%), finanzas (17.41 $\%)$, energía (13.07\%), industria (13.04\%), entre otros (13.22\%). Según las estadísticas del Ministerio de Energía y Minas en relación al empleo, en el año 2016 el sector minero generó un promedio anual de 174 mil 126 puestos de trabajo directo. Del total de empleos directos registrados; 61,873 fueron generados por las compañías mineras titulares de la actividad minera en el país; mientras que 112,253 puestos laborales fueron originados por las empresas contratistas mineras que brindan servicios a dichas compañías.

En relación a las actividades mineras y civiles, la perforación y voladura de rocas son inevitables en dichas actividades. Por lo tanto, el uso de explosivos es probablemente el medio más ampliamente utilizado para el fracturamiento de las rocas. Una mala fragmentación puede hacer necesario la realización de voladuras secundarias o bien, la necesidad de romper los tamaños mediante medios mecánicos, añadido al sobre costo que se supone. Un parámetro que va a definir el resultado de una voladura es la granulometría de la pila de un material volado y la manera más exacta de conocerlo, es mediante el índice de fragmentación medio $\mathrm{X}_{50}$, que se obtiene mediante diferentes técnicas, la más usual se determina mediante las distintas fracciones que ingresan a la planta de tratamiento, y que pueden ser separados por medio de sub procesos y mallas en las diferentes fracciones.

Los parámetros que afectan a la fragmentación de la roca se pueden clasificar en dos grupos: El primer grupo controla los pa-

1 Ingeniero de Minas, Consultor independiente en Seguridad, Salud Ocupacional, Medio Ambiente y Calidad. email: rojaslinares8@yahoo.com 
rámetros de diseño y los parámetros relacionados con explosivos; y el segundo son los parámetros incontrolables como las propiedades físicas (litología, mineralogía etc.) y geo-mecánicas de la roca.

En la predicción de la fragmentación de la roca, el primer paso para la optimización, son los parámetros de diseño para producir fragmentación necesaria del tamaño. Así mismo se han realizado varios estudios sobre los factores predictivos de la voladura en referencia a la fragmentación con parámetros controlables y no controlables.

- De acuerdo con Hustrulid (1999) afirma que la ingeniería de la fragmentación va a ser una parte importante de la minería en el futuro. A medida que las máquinas de carga y transporte se vuelven más automatizados, como las fajas transportadoras, será necesaria una especificación mucho mejor del tamaño y/o granulometría del material fragmentado.

- Así mismo, Esen (2013) refiere en su investigación de "Fragmentation Modelling and the Effects of ROM Fragmentation on Comminution Circuits", que la fragmentación también puede tener un impacto notable en la economía de la recuperación de lixiviación en pilas, como fue demostrado por (Sheikh \& Chung, 1987), en un estudio realizado en la Mina Denison, Ontario, Canadá. Llegaron a la conclusión que la viabilidad de lixiviación en pilas en esta

- operación depende de la alternativa de mantener las reservas de mineral roto $u$ fragmentado durante largos periodos de tiempo, por lo tanto, es necesario adoptar estrategias de optimización a través de la implementación de adecuados diseños de voladura.

- El Instituto Geológico y Minero de España (1987) sostiene que:

"El grado de fragmentación es un término genérico que se utiliza para definir la distribución granulométrica del material volado (...). Sobre la fragmentación influyen un gran número de variables controlables (...). No existe un método o fórmula de predicción exacta, pero en los últimos años un gran número de investigadores han trabajado en dicho campo llegando a modelos que van desde las simples fórmulas empíricas a complejas simulaciones con ordenadores" (p.366).
- Faramarzi, Farsangi y Mansouri (2013) en su investigación describe, que la fragmentación de rocas ha sido la preocupación de muchos trabajos de investigación debido a que es considerada como el aspecto más importante de la producción, ya que afecta a los costos de los trabajos de perforación, voladura y la eficiencia de todos los subsistemas, tales como; la carga, el transporte y otros de las operaciones mineras.

- Bagherpour, Monjezi, Sayadi y Yari (2015) sostienen que la voladura es una de las operaciones más importantes de los proyectos mineros, inapropiadamente la voladura patrón puede conducir a eventos no deseados como la mala fragmentación, ruptura de regreso, lanzamiento de rocas, etc. y pueden afectar a toda la operación física y económicamente.

El estudio del arte, lleva a nombrar a algunos personajes pioneros en la predicción de la fragmentación del material volado como. Larsson, Kuznetsov, Svedefo, Dinis da Gama, Cunningham Claude., que han realizado estudios y pruebas según el Instituto Geológico y Minero de España. (1987), Hustrulid (1999) y Holmberg (2005) que han servido de base para las nuevas investigaciones. Las siguientes investigaciones, demuestran la importancia que el mundo científico y académico, están realizando en la actualidad en referencia a la ingeniería de la fragmentación en los procesos de voladura de rocas.

- Kihlstrom y Langerfors (1976) introduce a los mecanismos de rotura de rocas con explosivos, para lo cual nos expone que el cálculo de la carga de los explosivos, en las voladuras de rocas es una función de las siguientes variables: Magnitudes geométricas del macizo rocoso, factores característicos de los explosivos y factores que dependen de la roca y del grado de fijación.

- Holdemberg, Lee y Persson (1993) tomando como base las teorías de existentes, desarrollan las técnicas de voladuras controladas de contorno, con tipos de recorte, demostrando que la fragmentación de las rocas está en función de la geometría del macizo rocoso, propiedades de las roca y características del material del explosivo, presentando nuevas constantes en la formulación descrita, relacionando la termodinámica del explosivo en la ingeniería de la fragmentación. Con el objetivo de obtener el contorno de la excavación con 
el menor daño posible y controlando la estructura del macizo, se han elaborado diferentes métodos que se agrupan bajo el nombre de explosión de contorno.

- Brown y Hock (1985) hacen una recopilación de las mejores técnicas de sostenimiento para el macizo rocoso en labores subterráneas y proponen el GSI como alternativa nueva, el conocimiento que transmite en su obra "Excavaciones Subterráneas en Roca", es básico para entender el macizo rocoso en sus detalles, como la rugosidad y el estado de las fisuras, el índice de calidad de roca, la dureza, la elasticidad etc., valuaciones que se toman en cuenta, para generar una óptima fragmentación el diseño de perforación y voladura de rocas.

- Singiresu (2009) sostiene que, en los últimos años, se han desarrollado algunos métodos de optimización que son conceptualmente diferente de las técnicas tradicionales de programación matemática. Estos métodos han sido etiquetados como los métodos modernos y no tradicionales de optimización. La mayoría de estos métodos se fundamentan en ciertas características y el comportamiento de factores biológicos, moleculares, enjambre de insectos y los sistemas neurobiológicos. De los cuales podemos nombrar los siguientes.

- Fisne, Hudaverdi y Kuzu (2012) realizan una investigación de la fragmentación de la voladura, usando el tamaño medio de la fragmentación y el índice de la fragmentación, en base a un análisis multivariante.

Huang, Shi, Wei, Wu y Zhou (2011) realizan un nuevo enfoque de análisis vectorial con el apoyo de máquinas, para predecir del tamaño medio de las partículas en la fragmentación rocas, en las voladuras superficiales.

Hudaverdi, Kulatilake, Kuzu y Qiong (2010) proponen un nuevo enfoque para la predicción del tamaño medio de las partículas en la fragmentación en la voladura de rocas, usando redes neuronales.

Saeid (2015) realiza una investigación de predicción de las voladuras, inducidas por las vibraciones del suelo, en base a una programación de algoritmos genéticos, contrastando con redes neuronales.

- La Escuela Técnica Superior de Ingenieros de Minas de Madrid (2013) define que los avances presentados en el diseño de las mallas para voladura, son avances desarrollados a partir múltiples pruebas realizadas y cuyos resultados son ajustados a ecuaciones matemáticas, por estos motivos es muy útil el conocer de forma previa, la granulometría de una pila de material volado. Así mismo establece que el objetivo de una voladura, es aquella en la que se buscan resultados óptimos en la fragmentación y un desplazamiento controlado del material. Para lograr este objetivo y evaluar el correcto rendimiento de una voladura, se deben tener en cuenta tres factores fundamentales, que son claves para un correcto diseño y control de las voladuras, las cuales son.

a) Una correcta cantidad de energía. b) Una correcta distribución de energía. c) Un correcto confinamiento de energía.

Así los tres factores están íntimamente relacionados entre sí, de modo que a la falta de uno de ellos, hace que el rendimiento obtenido no se corresponda con el objetivo deseado.

Continuando, describiremos los objetivos y la justificación del presente estudio, las cuales son :

Objetivo general : Es teorizar un nuevo enfoque predictivo de la fragmentación, en la voladura de rocas, aplicando técnicas de optimización.

\section{Objetivos específicos serán :}

- Determinar el promedio medio de la fragmentación X50, del proceso de voladura de rocas, mediante la teoría de Kuz-Ram.

- Determinar el promedio medio de la fragmentación X50, del proceso de voladura de rocas, mediante técnicas de Análisis Multivariante.

- Determinar una estadística descriptiva y una inferencia de análisis paramétrico de los datos.

- Evaluar los parámetros controlables como burden, espaciamiento entre los taladros, factor de carga etc., que ejercen una influencia significativa para la predicción de la fragmentación, en la voladura de rocas.

El estudio se justifica porque manejando el control de la fragmentación en los procesos de voladura, servirá optimizar los procesos operacionales mineros y metalúrgicos, y por ende, reducir los costos.

\section{MÉTODO}


El caso estudio toma los datos expuestos del Journal elsevier de Hudaverdi et al. (2010), que consta de 90 voladuras realizadas en diferentes centros mineros en Europa, los datos describen un primer grupo de parámetros de diseño de las voladuras, donde : (B) es el burden, (S) es el espaciamiento entre los taladros, $(T)$ es el taco, $(H)$ es la altura del banco, (D) es el diámetro del taladro, (Pf) es el factor de potencia, todas las voladuras fueran hechas usando ANFO y donde se usaron los siguientes ratios (S/B), (H/B), (B/D), (T/B).

Así mismo el segundo grupo de los parámetros que afectan a la fragmentación se han divido en dos subgrupos el primer subgrupo contiene las propiedades físicas y mecánicas de la roca intacta, mientras que el segundo subgrupo contiene las propiedades de las discontinuidades y la estructura del macizo rocoso, podemos describirlos a $(E)$ como el módulo de elasticidad que es usado para representar a las propiedades mecánicas de la roca, y a $(\mathrm{XB})$ que representa a la estructura in situ del macizo rocoso. La descripción de las minas donde se obtuvieron las datas son:

- Enusa, (En) es una mina de uranio a cielo abierto (Open Pit), Reocin (Rc) es una mina a cielo abierto y Reocin underground $(\mathrm{Ru})$ ambas explotan zinc, todas estas minas están localizadas en España.

- Murgul (Mg) es una mina de cobre, localizada al noreste de Turkia,

- Mrica (Mr) presenta una perfomance de las canteras Indonesia, la investigación fue hecha por SveDeFo (Swedish Detonic Reserch Foundation), Investigaciones hechas en predicción de modelos de la fragmentación.

- El símbolo (Sm), presenta una performance de una mina de carbón a cielo abierto (Open Pit), en la cuenca del soma localizada al occidente de Turquía

- Las voladuras indicadas por el símbolo (Db) es una perfomance de la mina de manganesio Dongri-Buzurg a cielo abierto (Open Pit), situada en el centro de India, generalmente el macizo rocoso fue de micas esquistosas y muscovitas esquistosas.

- Los símbolos de las voladuras de (Ad) y $(\mathrm{Oz})$ son una performance de las canteras Akdaglar y Ozmert de la cuenca de Cendere localizada al norte de Estanbul.
Cabe mencionar que dichas datas, fueron sometidas a los siguientes métodos, utilizando los softwares; Matlab R2015a, SPSS V22, y Minitab 17.

1ro. Al Modelo KUZ-RAM.

2do. A un Análisis Multivariante, (MVA)

3ro. A Redes Neuronales Artificiales (ANN).

El objetivo es encontrar teóricamente, mediante las técnicas pre-descritas, el índice medio del tamaño de las partículas (Indice de fragmentación teórica $X_{50}$ ), con la finalidad de que dicho resultado sea contrastado, con el índice de fragmentación real $\mathrm{X}_{50}$ (Que será un cálculo hecho en base a diferentes mallas en el proceso de molienda), y finalmente se podrá determinar, cuál de las tres técnicas teóricas, es la que tiene mayor afinidad a la fragmentación real, en otras palabras, se podría decir, cuál de las tres técnicas, es la más óptima.

Para plantear un nuevo enfoque de la fragmentación, el presente estudio toma las siguientes bases teóricas, que a continuación se describirán.

2.1. EI Modelo KUZ-RAM, según Instituto Geológico y Minero de España (1987), Holmberg (2005) y Hustrulid (1999) este modelo fue desarrollado por Cunningham Claude en el año de 1983, por el AECI en South Africa, tomando la curva de distribución de la granulometría de Rosin y Rammler en base a la ecuación empírica de V. M. Kuznetsov propuesta en el año de 1973.

\section{Las ecuaciones adaptadas y propuestas por Cunningham Claude fueron :}

$$
\begin{aligned}
& \bar{X}=A^{*}\left(\frac{V_{o}}{Q_{e}}\right)^{0.8} * Q_{e}{ }^{1 / 6} *\left(\frac{S_{A N F O}}{115}\right)^{-19 / 30} \quad \text { (Ec. 1) } \\
& \bar{X}=A(K)^{-0.8} Q_{e}^{1 / 6}\left(\frac{115}{S_{A N F O}}\right)^{19 / 30} \quad \text { (Ec. 2) }
\end{aligned}
$$

\section{Donde:}

X50 = Tamaño medio de los fragmentos de Voladura. $(\mathrm{cm})$

$\mathrm{A}=$ Factor de roca.

Vo $=$ Volumen de roca fragmentada por taladro $\left(\mathrm{m}^{3}\right)$

$\mathrm{Q}=$ Cantidad de TNT equivalente a la carga de explosivo por taladro

$\mathrm{Qe}=$ Kilos de explosivo por taladro.

$S$ Anfo = Potencia relativa en peso referida al Anfo

Cunningham $C$., indica que en tipos de rocas muy débiles $A=8$ y el límite superior es $A=12$; Una al- 
ternativa para cuantificar mejor la selección de "A", es utilizando el Índice de Volatibilidad propuesto inicialmente por Ather Lilly en 1986, en cual ha sido adaptado para esta aplicación por Cunningham C., en 1987. La ecuación es la siguiente:

$$
A=0.06 *(R M D+J F+R D I+H F)(E c .3)
$$

\section{Donde:}

RMR : Descriptor de la masa Rocosa.

JPS : Espaciamiento de las diaclasas verticales.

JPA : Angulo del plano de la diaclasa.

RDI : Influencia de la densidad.

HF : Factor de dureza.

Según Hustrulid (1999) dicho modelo toma los Indices de la Fragmentación de (Ather Lily, 1986) y de la Escala de dureza de Mohs (Roberts, 1977). Para proponer el Índice de Factor "A".

2.2. ANÁLISIS MULTIVARIANTE : En el presente análisis metodológico se tomó como base a los siguientes autores:

Baillo y Grane (2008), De la Vara y Gutiérrez (2012), Montgomery (2002), Montgomery y Runger (2002) y Spiegel (1961). Luego realizar un extracto de las diferentes técnicas de dichos autores, seguidamente se reordeno la data, a una forma matricial, pasando luego ser sometida a un Análisis Multivariante (MVA). Los test más relevantes que se hicieron fueron:

a. Analisis de Fiabilidad (Alfa de Cronbach).

b. Prueba de KMO, Bartlett y Hoteling.

c. Análisis de Varianzas y Covarianzas

d. Análisis de la matriz de dispersión

e. Análisis Box Plot

f. Análisis de Regresiones y Correlaciones Multivariantes

\section{g. Análisis de Conglomerados.}

El análisis de varianzas (ANOVA), es un tratamiento en función a probabilidades, la finalidad de dicho análisis es rechazar la hipótesis nula $(\mathrm{Ho})$, y aceptar la hipótesis alternativa (Ha). El tratamiento se confirma, si el resultado del $p$-valor calculado, es menor que el $(p-s i g=0.05)$ de contraste. Por lo tanto, si eso se cumple, es posible hacer un buen análisis inferencial con una probabilidad del (95\%).
2.3. REDES NEURONALES. Según Kriesel (2005) las componentes de una red neuronal, técnicamente consisten en unidades de procesamiento simples de neuronas y conexiones ponderadas dirigidas entre esas neuronas, aquí el esfuerzo de una conexión (o las conexiones de los pesos) entre dos neuronas i y j se conoce como Wij. (p.3).

Así mismo para Basogain (1998)

"En las Redes Neuronales Artificiales, ANN, la unidad análoga a la neurona biológica es el elemento procesador, PE (Process Element). Un elemento procesador tiene varias entradas y las combina normalmente con una suma básica. La suma de las entradas es modificada por una función de transferencia y el valor de la salida de esta función de transferencia se pasa directamente a la salida del elemento procesador. La salida del PE se puede conectar a las entradas de otras Neuronas Artificiales (PE) mediante conexiones ponderadas correspondientes a la eficacia de la sinapsis de las conexiones neuronales (...). El interés de las ANN no reside solamente en el modelo del elemento PE sino en las formas en que se conectan estos elementos procesadores. Generalmente los elementos PE están organizados en grupos llamados niveles o capas. Una red típica consiste en una secuencia de capas con conexiones entre capas adyacentes consecutivas. Existen dos capas con conexiones con el mundo exterior. Una capa de entrada, buffer de entrada, donde se presentan los datos a la red y una capa buffer de salida que mantiene la respuesta de la red a una entrada. El resto de las capas reciben el nombre de capas ocultas"(p.3).

La aplicación de Redes Neuronales Artificiales (ANN), es quizás hoy en día la arquitectura de red que contiene los aspectos más destacados de la teoría de Redes Neuronales, simple en su estructura y clara en el sentido matemático.

Para el presente caso se aplicará una red neuronal de tipo multicapa, con 7 neuronas en la 1 ra capa que procesaran las datas de: $S / B, H / B, B / D$, $\mathrm{T} / \mathrm{B}, \mathrm{Pf}, \mathrm{XB}, \mathrm{E} ; 14$ neuronas en la 2 da capa y 1 neurona en la capa de salida, por consiguiente, el resultado será un X50 teórico, que puede contrastarse con el X50 real expuesto en la tabla 1. Así mismo, en la figura 1 se presenta la estructura del modelo de la red relacionada a la data de ingreso, pesos sinápticos y las bias, seguidamente en las 


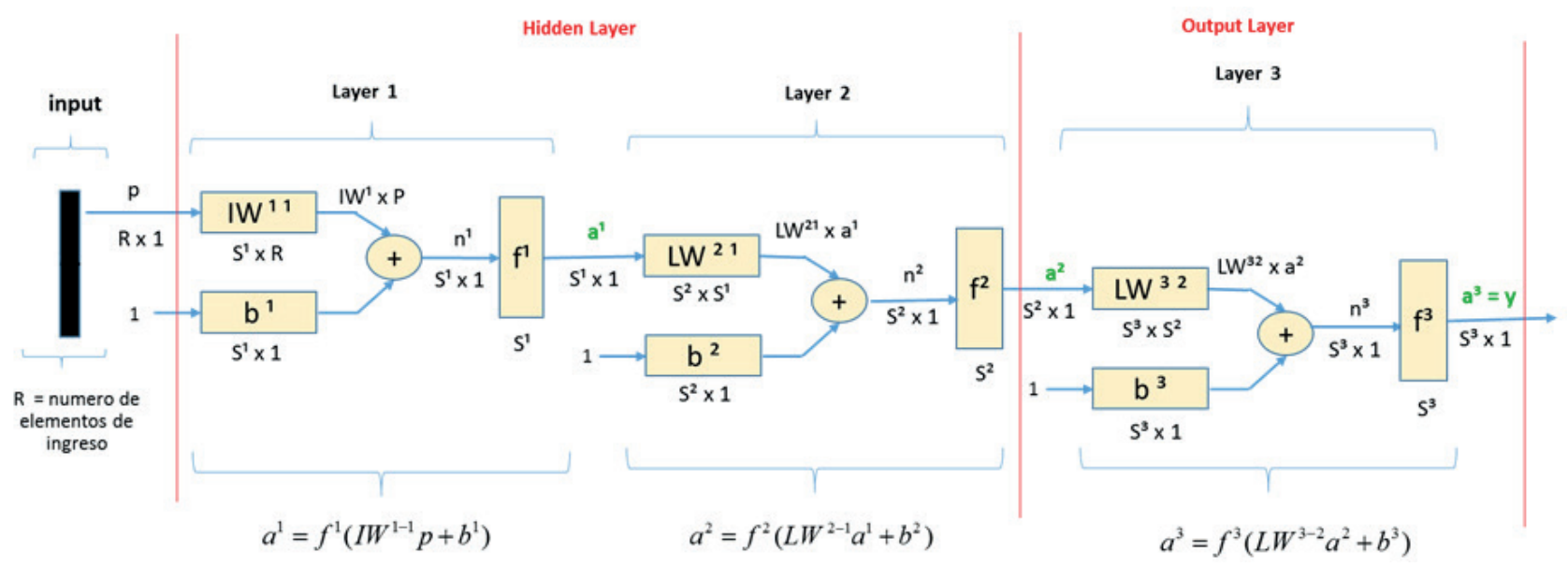

Figura 1. Estructura del Modelo Neuronal Multicapa

Fuente: Elaboración propia

Estructura de la función general $a^{3}=f^{3}\left(L W^{3-2} f^{2}\left(L W^{2-1} f^{1}\left(I W^{1-1} p+b^{1}\right)+b^{2}\right)+b^{3}\right)=y \quad$ (Ec. 4)

Donde: $s^{1}, s^{2}, s^{3} \quad$ Numero de neuronas en la capa 1, en la capa 2, y respectivamente en la capa 3.

$I W^{1-1} \quad$ Pesos Sinápticos de la matriz, para conectarse del ingreso a la capa 1.

$L W^{2-1} \quad$ Pesos Sinápticos de la matriz, para conectarse de la capa 1 a la capa 2.

$L W^{3-2} \quad$ Pesos Sinápticos de la matriz, para conectarse de la capa 2 a la capa 3.

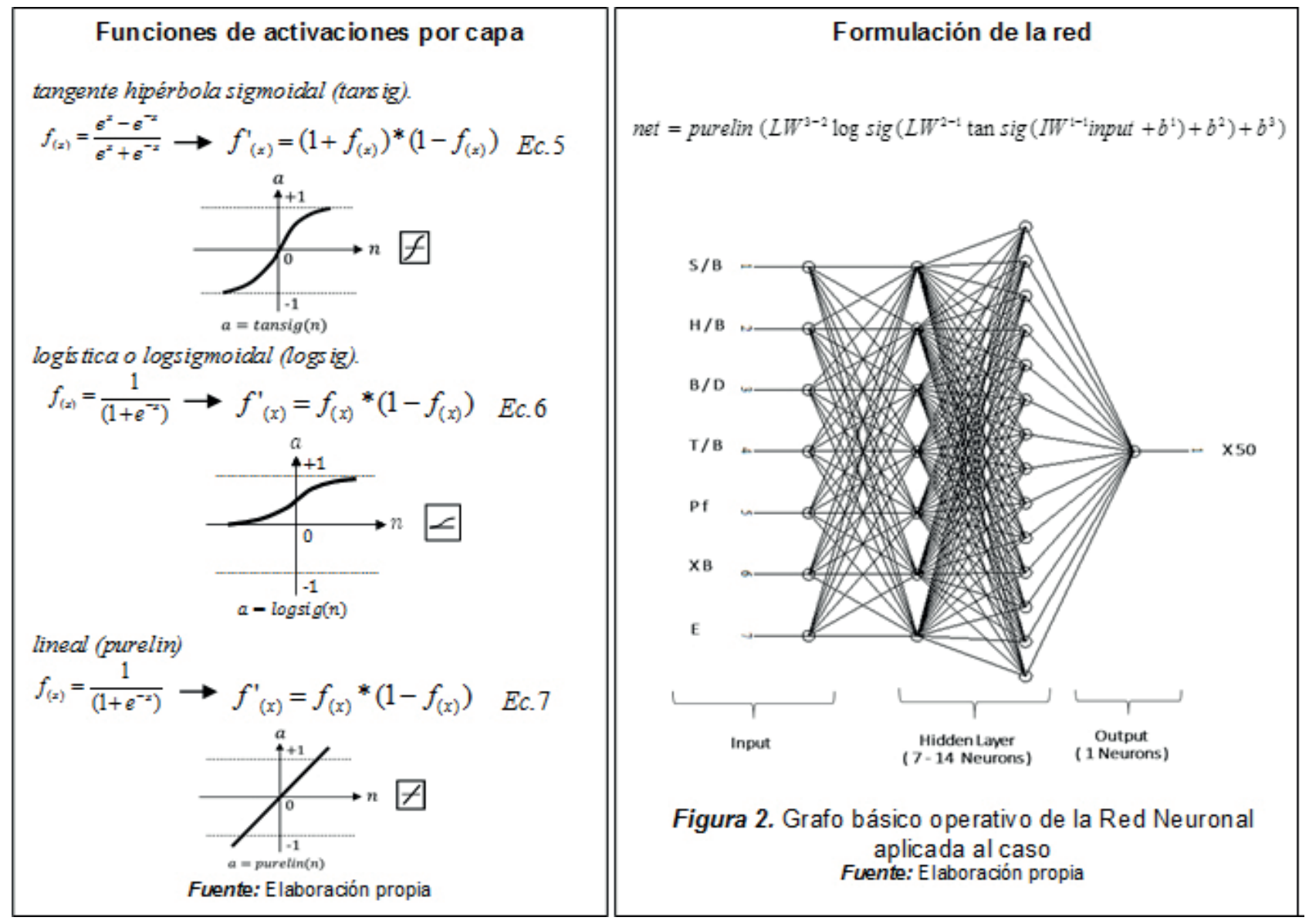


ecuaciones 5,6 y 7 presentamos las funciones de activación y en la figura 2 , el grafo básico operativo de la Red Neuronal.

\section{RESULTADOS Y DISCUSIONES}

En las siguientes líneas, se presentan los resultados de las pruebas de las estadísticas descriptivas en referencia a las datas tratadas, los resultados obtenidos nos brindaran una información en referencia, a que si las datas son fiables o no son fiables, paso a continuación a presentar los resultados

INTERPRETACION 1: La prueba del analisis de varianzas (ANOVA), con su valor sig (2,2637e-190), confirma el rechazo de la Hipotesis Nula $(\mathrm{H} 0)$ y por lo tanto se acepta la hipotesis alternativa $(\mathrm{H} 1)$, confirmado si es factible hacer un analisis inferencial (con una probabilidad al 95\%), se podria decir que las datas tienen corelacion, asi mismo el resultado de levene confirma lo esperado.

INTERPRETACIÓN 2: El presente grafico de la figura 3 del diagrama de caja, nos especifica que el parámetro $(E)$ es la data que tiene la mayor variabilidad, donde el cuartil (Q2-Q3), mantiene una mayor dispersión de datos que el cuartil (Q1-Q2).

Continuando podemos apreciar que el parámetro $(B / D)$ es la que tiene la mayor mediana que todos los parámetros, así mismo este nos presenta datos

\section{Prueba de homogeneidad de varianzas}

\begin{tabular}{|l|c|c|c|}
\hline Indicadores \\
\hline Estadístico de Levene & df1 & df2 & Sig. \\
\hline 315,476 & 6 & 623 &, 000 \\
\hline
\end{tabular}

\begin{tabular}{|l|r|r|r|r|r|}
\multicolumn{7}{c|}{$\begin{array}{c}\text { ANOVA } \\
\text { Indicadores }\end{array}$} \\
\cline { 2 - 7 } & Suma de cuadrados & gl & Media cuadrática & F & Sig. \\
\hline Entre grupos & 97291,562 & 6 & 16215,260 & 332,130 & $2,2637 \mathrm{e}-190$ \\
\hline Dentro de grupos & 30416,103 & 623 & 48,822 & & \\
\hline Total & 127707,665 & 629 & & & \\
\hline
\end{tabular}

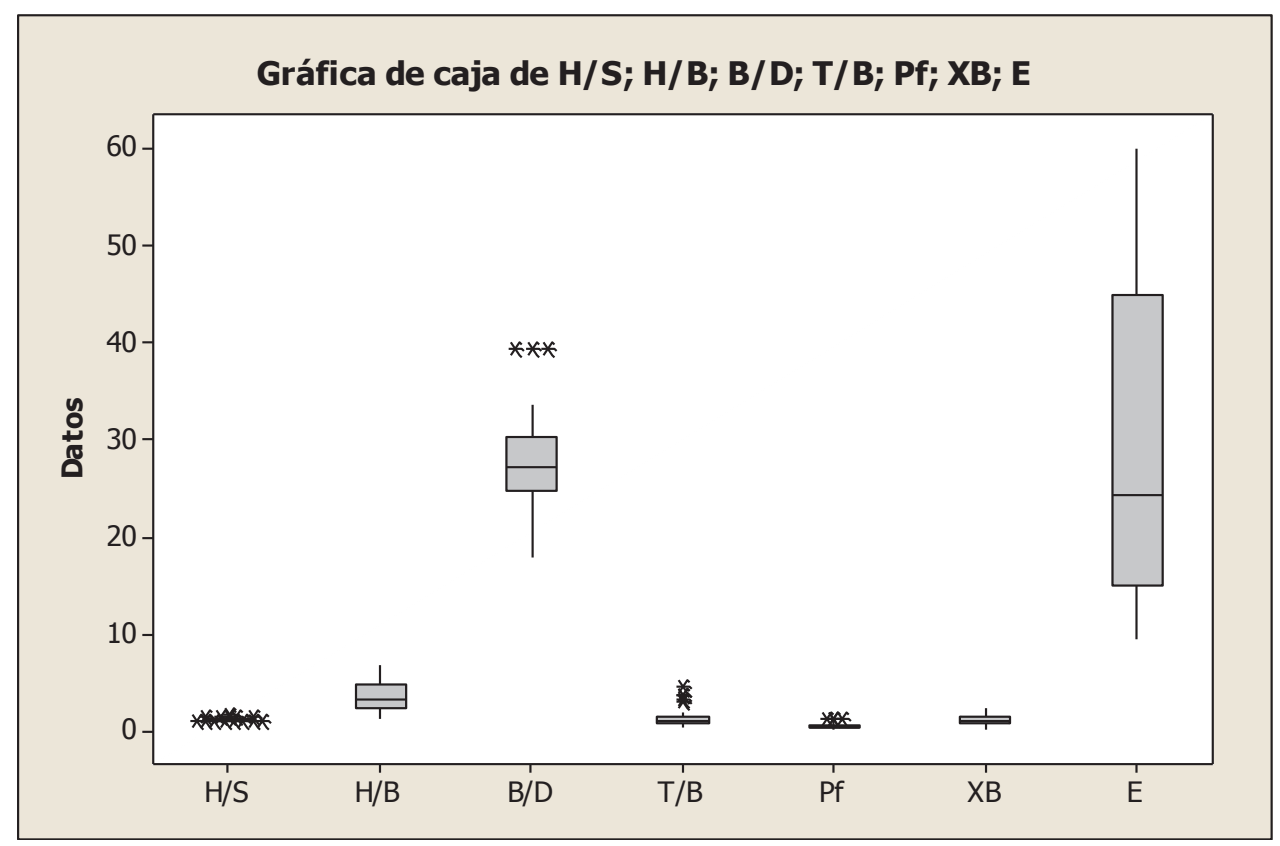

Figura 3. Diagrama de caja

Fuente: Elaboración propia 
atípicos muy dispersos superiores al cuartil (Q3), se puede resumir apreciando el grafico que, de estos dos grupos, sus datas poseen la mayor variabilidad u dispersión, que todos los demás parámetros. Así mismo los demás parámetros como $(H / S),(H / B)$, (T/B), (Pf), (XB), poseen una dispersión mínima de sus datos.

Tabla 1. Comparación de resultados de $X_{50}$ estimados por diferentes métodos

\begin{tabular}{|c|c|c|c|c|c|}
\hline No. & Tajo & $\begin{array}{c}\mathbf{X}_{50} \\
\text { KUZ-RAM }\end{array}$ & $\begin{array}{c}\mathbf{X}_{50} \\
\text { MVAR }\end{array}$ & $\begin{array}{c}\mathbf{X}_{50} \\
\text { ANN }\end{array}$ & $\begin{array}{c}\mathbf{X}_{50} \\
\text { real (m) }\end{array}$ \\
\hline $\mathbf{1}$ & En1 & 0.401 & 0.367 & 0.37 & 0.37 \\
\hline $\mathbf{2}$ & En2 & 0.401 & 0.367 & 0.37 & 0.37 \\
\hline $\mathbf{3}$ & En3 & 0.401 & 0.401 & 0.395 & 0.33 \\
\hline $\mathbf{4}$ & En4 & 0.401 & 0.403 & 0.42 & 0.42 \\
\hline $\mathbf{5}$ & En5 & 0.401 & 0.401 & 0.395 & 0.46 \\
\hline $\mathbf{6}$ & En6 & 0.577 & 0.535 & 0.37 & 0.37 \\
\hline $\mathbf{7}$ & En7 & 0.508 & 0.497 & 0.64 & 0.64 \\
\hline $\mathbf{8}$ & En8 & 0.443 & 0.448 & 0.34 & 0.42 \\
\hline $\mathbf{9}$ & En9 & 0.443 & 0.448 & 0.34 & 0.26 \\
\hline $\mathbf{1 0}$ & En10 & 0.481 & 0.477 & 0.42 & 0.42 \\
\hline $\mathbf{1 1}$ & En11 & 0.508 & 0.499 & 0.345 & 0.31 \\
\hline $\mathbf{1 2}$ & En12 & 0.508 & 0.499 & 0.345 & 0.38 \\
\hline $\mathbf{1 3}$ & Rc2 & 0.63 & 0.47 & 0.48 & 0.48 \\
\hline $\mathbf{1 4}$ & Rc3 & 0.664 & 0.488 & 0.48 & 0.48 \\
\hline $\mathbf{1 5}$ & Rc4 & 0.609 & 0.525 & 0.75 & 0.75 \\
\hline $\mathbf{1 6}$ & Rc5 & 0.66 & 0.551 & 0.96 & 0.96 \\
\hline $\mathbf{1 7}$ & Rc6 & 0.576 & 0.505 & 0.76 & 0.76 \\
\hline $\mathbf{1 8}$ & Rc7 & 0.628 & 0.551 & 0.53 & 0.53 \\
\hline $\mathbf{1 9}$ & Rc8 & 0.651 & 0.565 & 0.56 & 0.56 \\
\hline $\mathbf{2 0}$ & Rc9 & 0.625 & 0.551 & 0.74 & 0.74 \\
\hline $\mathbf{2 1}$ & Rc10 & 0.591 & 0.532 & 0.44 & 0.44 \\
\hline $\mathbf{2 2}$ & Mg1 & 0.328 & 0.277 & 0.23 & 0.23 \\
\hline $\mathbf{2 3}$ & Mg2 & 0.328 & 0.273 & 0.25 & 0.25 \\
\hline $\mathbf{2 4}$ & Mg3 & 0.387 & 0.295 & 0.27 & 0.27 \\
\hline $\mathbf{2 5}$ & Mg4 & 0.387 & 0.276 & 0.3 & 0.3 \\
\hline $\mathbf{2 6}$ & Mg5 & 0.419 & 0.311 & 0.38 & 0.38 \\
\hline $\mathbf{2 7}$ & Mg6 & 0.419 & 0.315 & 0.37 & 0.37 \\
\hline $\mathbf{2 8}$ & Mg7 & 0.419 & 0.321 & 0.38 & 0.38 \\
\hline $\mathbf{2 9}$ & Ru1 & 0.92 & 0.371 & 0.64 & 0.64 \\
\hline $\mathbf{3 0}$ & Ru2 & 0.893 & 0.627 & 0.54 & 0.54 \\
\hline $\mathbf{3 1}$ & Ru3 & 0.893 & 0.629 & 0.51 & 0.51 \\
\hline $\mathbf{3 2}$ & Ru4 & 1.087 & 0.751 & 0.64 & 0.64 \\
\hline $\mathbf{3 3}$ & Ru5 & 0.81 & 0.554 & 0.54 & 0.54 \\
\hline $\mathbf{3 4}$ & Ru6 & 0.825 & 0.568 & 0.69 & 0.69 \\
\hline $\mathbf{3 5}$ & Mr1 & 0.377 & 0.218 & 0.15 & 0.17 \\
\hline $\mathbf{3 6}$ & Mr2 & 0.368 & 0.208 & 0.17 & 0.17 \\
\hline $\mathbf{3 7}$ & Mr3 & 0.377 & 0.218 & 0.15 & 0.13 \\
\hline 38 & Mr4 & 0.363 & 0.203 & 0.17 & 0.17 \\
\hline 39 & Mr5 & 0.416 & 0.251 & 0.13 & 0.13 \\
\hline $\mathbf{4 0}$ & Mr6 & 0.47 & 0.233 & 0.15 & 0.15 \\
\hline & & & & & \\
\hline
\end{tabular}

\begin{tabular}{|c|c|c|c|c|c|}
\hline No. & Tajo & $\begin{array}{c}\mathrm{X}_{50} \\
\text { KUZ-RAM }\end{array}$ & $\begin{array}{c}X_{50} \\
\text { MVAR }\end{array}$ & $\begin{array}{c}X_{50} \\
\text { ANN }\end{array}$ & $\begin{array}{c}X_{50} \\
\text { real }(m)\end{array}$ \\
\hline 41 & Mr7 & 0.347 & 0.123 & 0.18 & 0.18 \\
\hline 42 & Mr8 & 0.528 & 0.2 & 0.19 & 0.19 \\
\hline 43 & Mr9 & 0.479 & 0.176 & 0.16 & 0.16 \\
\hline 44 & Mr10 & 0.46 & 0.088 & 0.17 & 0.17 \\
\hline 45 & Mr11 & 0.522 & 0.122 & 0.21 & 0.21 \\
\hline 46 & Db1 & 0.241 & 0.3 & 0.44 & 0.44 \\
\hline 47 & Db2 & 0.264 & 0.305 & 0.76 & 0.76 \\
\hline 48 & Db3 & 0.265 & 0.325 & 0.35 & 0.35 \\
\hline 49 & Db4 & 0.317 & 0.311 & 0.55 & 0.55 \\
\hline 50 & Db5 & 0.399 & 0.339 & 0.35 & 0.35 \\
\hline 51 & Db6 & 0.42 & 0.484 & 0.23 & 0.23 \\
\hline 52 & Db7 & 0.353 & 0.43 & 0.4 & 0.4 \\
\hline 53 & Db8 & 0.461 & 0.375 & 0.35 & 0.35 \\
\hline 54 & Db9 & 0.466 & 0.31 & 0.29 & 0.29 \\
\hline 55 & Sm1 & 0.561 & 0.194 & 0.19 & 0.15 \\
\hline 56 & Sm2 & 0.561 & 0.194 & 0.19 & 0.19 \\
\hline 57 & Sm3 & 0.561 & 0.194 & 0.19 & 0.23 \\
\hline 58 & Sm4 & 0.561 & 0.263 & 0.25 & 0.22 \\
\hline 59 & Sm5 & 0.561 & 0.263 & 0.25 & 0.24 \\
\hline 60 & Sm6 & 0.561 & 0.263 & 0.25 & 0.26 \\
\hline 61 & Sm7 & 0.561 & 0.263 & 0.25 & 0.28 \\
\hline 62 & Ad1 & 1.018 & 0.191 & 0.15 & 0.15 \\
\hline 63 & Ad2 & 0.952 & 0.136 & 0.17 & 0.17 \\
\hline 64 & Ad3 & 0.901 & 0.088 & 0.14 & 0.14 \\
\hline 65 & Ad4 & 1.115 & 0.273 & 0.16 & 0.16 \\
\hline 66 & Ad5 & 0.806 & 0.189 & 0.21 & 0.21 \\
\hline 67 & Ad6 & 0.808 & 0.224 & 0.21 & 0.21 \\
\hline 68 & Ad7 & 0.75 & 0.229 & 0.15 & 0.15 \\
\hline 69 & Ad8 & 0.767 & 0.236 & 0.19 & 0.19 \\
\hline 70 & Ad9 & 0.743 & 0.269 & 0.18 & 0.18 \\
\hline 71 & Ad10 & 0.743 & 0.239 & 0.15 & 0.15 \\
\hline 72 & Ad11 & 0.803 & 0.209 & 0.14 & 0.14 \\
\hline 73 & Ad12 & 0.803 & 0.302 & 0.2 & 0.2 \\
\hline 74 & Ad13 & 0.976 & 0.233 & 0.15 & 0.15 \\
\hline 75 & Ad14 & 0.913 & 0.245 & 0.14 & 0.14 \\
\hline 76 & Ad15 & 0.601 & 0.168 & 0.15 & 0.15 \\
\hline 77 & Ad16 & 0.673 & 0.303 & 0.15 & 0.15 \\
\hline 78 & Ad17 & 0.493 & 0.138 & 0.19 & 0.19 \\
\hline 79 & Ad18 & 0.481 & 0.135 & 0.15 & 0.15 \\
\hline 80 & Ad19 & 0.481 & 0.186 & 0.17 & 0.17 \\
\hline 81 & Ad20 & 0.536 & 0.211 & 0.19 & 0.19 \\
\hline 82 & Ad21 & 0.508 & 0.166 & 0.22 & 0.22 \\
\hline 83 & Ad22 & 0.623 & 0.224 & 0.2 & 0.2 \\
\hline 84 & Oz1 & 1.133 & 0.144 & 0.27 & 0.27 \\
\hline 85 & $\mathrm{Oz2}$ & 0.974 & 0.208 & 0.14 & 0.14 \\
\hline 86 & $\mathrm{Oz3}$ & 0.974 & 0.179 & 0.14 & 0.14 \\
\hline 87 & Oz4 & 0.775 & 0.208 & 0.2 & 0.2 \\
\hline 88 & Oz5 & 1.139 & 0.193 & 0.17 & 0.17 \\
\hline 89 & Oz6 & 1.088 & 0.262 & 0.3 & 0.3 \\
\hline
\end{tabular}




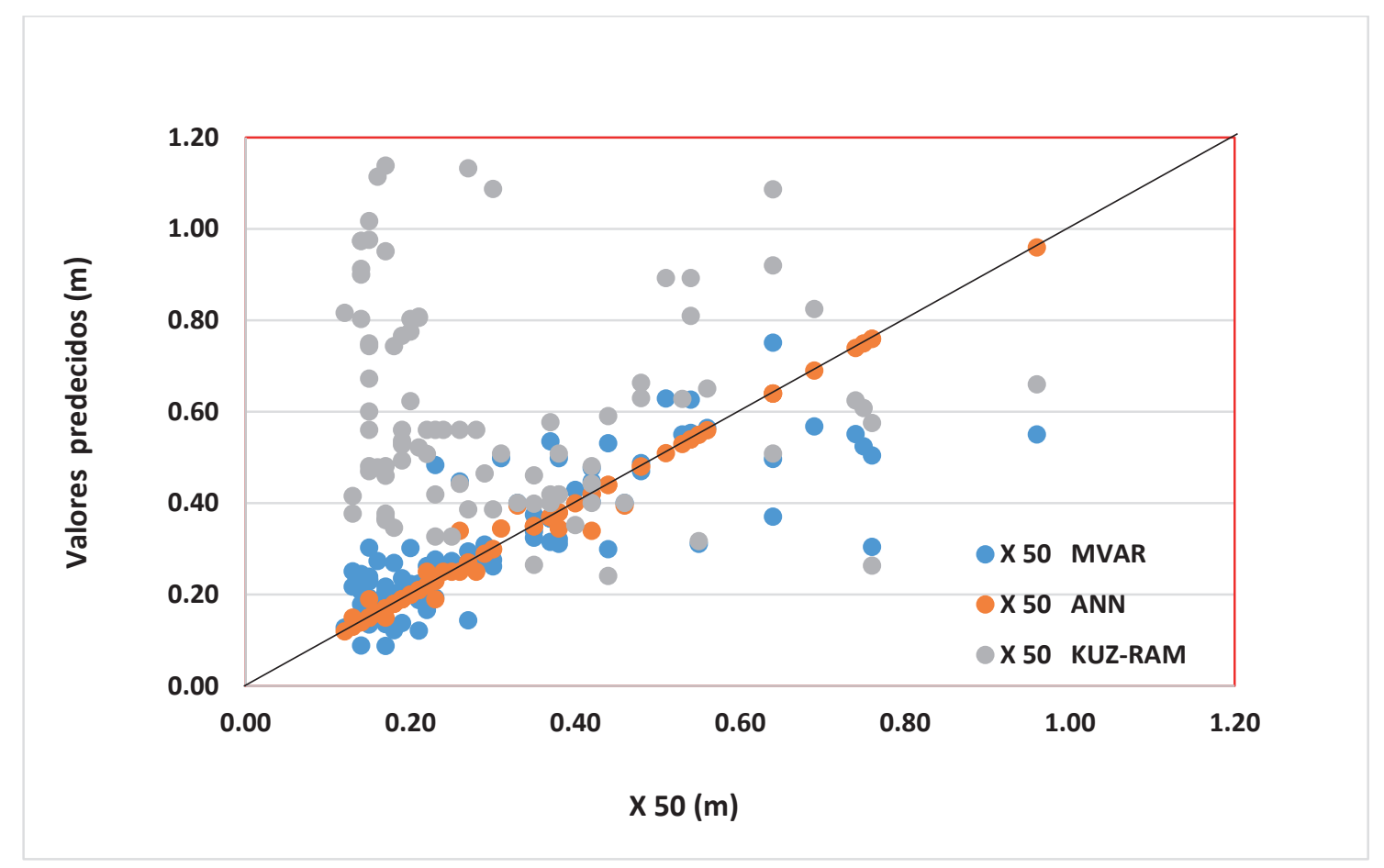

Figura 4. Ploteo

\begin{tabular}{|c|c|c|c|c|c|}
\hline No. & Tajo & $\begin{array}{c}\mathbf{X}_{50} \\
\text { KUZ-RAM }\end{array}$ & $\begin{array}{c}\mathbf{X}_{50} \\
\text { MVAR }\end{array}$ & $\begin{array}{c}\mathbf{X}_{50} \\
\text { ANN }\end{array}$ & $\begin{array}{c}\mathbf{X}_{50} \\
\text { real }(\mathbf{m})\end{array}$ \\
\hline $\mathbf{9 0}$ & Oz7 & 0.817 & 0.128 & 0.12 & 0.12 \\
\hline
\end{tabular}

Fuente: Elaboración propia

INTERPRETACIÓN 3 : La tabla 1, presenta los resultados $\mathrm{X}_{50}$ estimados por los tres modelos; Kuz-Ram, Regresiones por Análisis Multivariante (MVAR) y Redes Neuronales Artificiales (ANN), mientras que la figura 4, podemos visualizar el mejor ajuste lineal de los tres métodos.

Tabla 2. Resumen de Indices de la performance de $R^{2}$

\begin{tabular}{|l|l|l|}
\hline \multicolumn{1}{|c|}{ Método } & \multicolumn{1}{c|}{ Ajuste lineal } & \multicolumn{1}{c|}{$\mathbf{R}^{2}$} \\
\hline KUZ-RAM & $y=0.0992 x+0.6362$ & 0.0065 \\
\hline MVRA & $Y=0.6246 x+0.1185$ & 0.6246 \\
\hline ANN & $y=0.9903 x+0.031$ & 0.9903 \\
\hline
\end{tabular}

\section{CONCLUSIONES}

1. Para el presente estudio comparativo se ha utilizado los tipos ajustes lineales, presentados en la tabla 2.

2. De los resultados $X_{50}$ estimados por los tres métodos, podemos decir que el mejor modelo de aproximación a la fragmentación real $\left(X_{50}\right)$, es mediante Redes Neuronales Artificiales (ANN) por presentar un mejor coeficiente determinación en su ajuste lineal $\left(R^{2}=0.9903\right)$ y el método que más dista en relación a la fragmentación real $\mathrm{X}_{50}$, es obtenido por el modelo KUZ-RAM debido a que presenta un coeficiente de ajuste lineal $\left(R^{2}=0.0065\right)$.

3. Para el caso de la aplicación mediante la técnica de regresiones en base a un análisis multivariante (MVAR), podemos decir por sus resultados, que nos da un ajuste lineal moderado.

4. Desarrollados los tres métodos, se concluye que el nuevo enfoque, delinea la técnica más óptima a utilizar para la predicción de la fragmentación.

\section{REFERENCIAS BIBLIOGRÁFICAS}

[1] Baillo, M.A. y Grane, Ch.A. (2008). 100 Problemas Resueltos de Estadística Multivariante Implementado en Matlab. Madrid, España: Delta.

[2] Bagherpour, R., Monjezi, M., Sayadi, A.R. y Yari, M. (2015). Blasting Operation Management Using Mathematical Methods. Engineering Geology for Society and Territory. 1, 483-493. 
[3] Basogain, O.X. (2008). Redes Neuronales Artificiales y sus Aplicaciones. Bilbao, España : Escuela Superior de Ingeniería de Bilbao, Dpto. de Ingenieria de Sistemas y Automatica.

[4] Brown, E.T. y Hoek, E. (1985). Excavaciones Subterráneas en Roca. Mexico D.F., Mexico: Mc Graw Hill.

[5] De la Vara, S.R. y Gutiérrez, P.H. (2012). Análisis y Diseño de Experimentos. Mexico D.F., Mexico: Mc Graw Hill.

[6] Escuela Técnica Superior de Ingenieros de Minas de Madrid (2013). Perforación y Voladura de Rocas en Minería. Madrid, España: Universidad Politecnica de Madrid.

[7] Esen, S. (2013). Fragmentation Modelling and the Effects of ROM Fragmentation on Comminution Circuits. International Mining Congress \& Extibition of Turkey, 23rd, 251-260

[8] Faramarzi, F., Farsangi, E. y Mansouri, H. (2013). A Rock Engineering Systems Based Model to Predict Rock Fragmentation by Blasting. International Journal of Rock Mechanics \& Mining Sciences, 60, 82-94.

[9] Fisne, A., Hudaverdy, T. y Kuzu, C. (2012). Investigation of the blast fragmentation using the mean fragment size and fragmentation index. International Journal of Rock Mechanics \& Mining Sciences, 56, 136-145.

[10] Holmberg, R. (2005). The Kuz-Ram Fragmentation model 20 years on C.V.B. Cunnigham. European Federation of Explosives Engineers, ISBN 0-9550290-0-7, 201-210.

[11] Holdemberg, R., Lee, J. y Persson, A. (1993). Rock Blasting and Explosive Engineering. Boca Ranton, London, U.K.: C.R.C. Press L.L.C.

[12] Huang, D., Shi, X. Z., Wei, W., Wu, B.B. y Zhou, J. (2012). Support vector machines approach to mean particle size of rock fragmentation due to bench blasting prediction. Transactions of Nonferrous Metals Society of China, 22, 432 441. Recuperado de https://www.scienciedirect. com/sciencie/article/pii/S1003632611611953
[13] Hudaverdi, T., Kulatilake, P., Kuzu, C. y Qiong, W. (2010). Mean particle size prediction in rock blast fragmentation using neural networks. Engineering Geology, 114, 298-311. Recuperado de https://www.scienciedirect. com/sciencie/article/pii/S00113795210000827

[14] Hustrulid, W. (1999). Blasting Principles for Open Pit Mining. Golden, Colorado, U.S.A. : A.A. Balkema, Rotterdam, Naherlands.

[15] Instituto Geológico y Minero de España (1987). Manual de Perforación y Voladura de Rocas. Madrid, España: IGME Etimsa.

[16] Kihlstrom, B. y Langerfors, U. (1976). Tecnica Moderna de Voladura de Rocas. Bilbao, España: Espartero.

[17] Kriesel, D. (2005). A Brief Introduction to Neural Network. Boon, Germany: University of Bonn.

[18] Ministerio de Energía y Minas del Perú. (2016). Anuario Minero (1). Recuperado de http: // www.minem.gob.pe.

[19] Montgomery, D.C. (2002). Diseño y Análisis de Experimentos. México D.F., Mexico: Limusa Wiley.

[20] Montgomery, D.C. y Runger, G.C. (2002). Probabilidad y Estadística Aplicada a la Ingeniería. México D.F., Mexico: Limusa Wiley.

[21] Singiresu, S. R. (2009). Engineering Optimization. New Jersey, U.S.A.: John Wiley \& Sons.

[22] Spiegel, M.R. (1961). Estadística. Mexico D.F., Mexico: Mc Graw-Hill.

[23] Saeid, R. D. (2015). Prediction of Blast Induced Ground Vibrations via Genetic Programming. International Journal of Mining Sciencie and Technology, 25, 1011-1015.

\section{Glosario \\ Open Pit "Tajo"}

